\title{
Inhibition of Zika Virus Replication by Synthetic Bis-Naphthoquinones
}

\author{
Daniel T. G. Gonzaga, ${ }^{a, b}$ Rafaela S. P. Gomes, ${ }^{c}$ Roberta K. F. Marra, ${ }^{a}$ Fernando C. da Silva, ${ }^{\circledR a}$ \\ Max W. L. Gomes, ${ }^{c}$ Davis F. Ferreira, ${ }^{d, e}$ Raissa M. A. Santos, ${ }^{a}$ Ana M. V. Pinto, ${ }^{f}$ \\ Norman Arthur Ratcliffe, ${ }^{g}$ Claudio C. Cirne-Santos, ${ }^{c}$ Caroline S. Barros, ${ }^{c}$ Vitor F. Ferreira ${ }^{*, a}$ \\ and Izabel Christina N. P. Paixã̃o ${ }^{\circledR} *, c$
}

\author{
${ }^{a}$ Instituto de Química, Universidade Federal Fluminense, 24020-141 Niterói-RJ, Brazil \\ ${ }^{b}$ Instituto Biomédico, Centro Universitário Estadual da Zona Oeste, 23070-200 Rio de Janeiro-RJ, Brazil \\ 'Instituto de Biologia, Universidade Federal Fluminense, 24020-141 Niterói-RJ, Brazil \\ ${ }^{d}$ Instituto de Microbiologia Prof. Paulo de Góes, Universidade Federal do Rio de Janeiro, \\ 21941-599 Rio de Janeiro-RJ, Brazil \\ ${ }^{e}$ Department of Molecular and Structural Biochemistry, North Carolina State University, \\ 120 W Broughton Dr, Raleigh, 27607 NC, USA \\ ${ }^{f}$ Unidade de Farmácia, Universidade Federal Fluminense, 24210-130 Niterói-RJ, Brazil \\ ${ }^{8}$ Department of Biociences, College of Science Swansea University, SA2 8PP Swansea, UK
}

\begin{abstract}
Zika virus (ZIKV) is a mosquito-borne pathogen which is a current global public health concern. There are currently no approved vaccines or antivirals against ZIKV infection. Taking into account that naphthoquinones have shown promising antiviral activity, the aim of this study was to describe the screening of two bis-naphthoquinones series against ZIKV. Twenty seven compounds were evaluated against ZIKV using Vero cells. The findings showed that among the compounds analyzed four were promising. Compound 3,3'-((2-nitrophenyl)methylene)bis(2-hydroxynaphthalene1,4-dione) containing the nitro group at the ortho position showed the best selectivity index, followed by compound 3,3'-(4-chlorophenylmethylene)bis(naphthalene-1,2,4-triyl triacetate) with the chlorophenylmethylene radical. These results demonstrate that these bis-naphthoquinones are largely effective in inhibiting the replication of ZIKV.
\end{abstract}

Keywords: Zika virus, Flavivirus, arbovirus, antiviral, bis-naphthoquinones

\section{Introduction}

The spread of the disease caused by the Zika virus (ZIKV) has become a serious public health problem of global proportions. The disease is transmitted to people through the bite of infected mosquitoes, Aedes aegypti and Aedes albopictus. ${ }^{1}$ Usually, the disease has moderate effects and symptoms disappear within a few days. Many people have no or only mild symptoms. However, ZIKV infection during pregnancy can cause a severe malformation of the fetus, known as microcephaly, as well as other serious brain damage. ${ }^{2,3}$ Some recent research ${ }^{4}$ also suggests that Guillain-Barré Syndrome, an uncommon sickness of the nervous system, is also associated with Zika virus infection.

*e-mail: izabeluff@gmail.com, vitorferreira@id.uff.br
ZIKV belongs to the virus family Flaviviridae and its name derives from the Zika Forest of Uganda, where the virus was first isolated in 1947 from a Rhesus macaque. ${ }^{5} \mathrm{In}$ recent years, ZIKV has been rapidly spreading in tropical South America and French Polynesia despite attempts to stop the spread of infection vectors. At present, Brazil is the country with the highest number of reported cases of infection, as well as of cases of microencephaly. ${ }^{6,7}$

In the process of controlling the spread of the disease caused by the virus, there are several approaches ranging from mosquito control, ${ }^{8}$ to interrupt the chain of transmission, to attacking the virus via multiple therapeutic agents. Vaccine development also has great potential but so far there are limited prospects of vaccine production in the short term. The Butantan Institute in Brazil in association with National Institutes of Health (NIH) has announced 
<smiles>[R]CCN(CC)CCCC(C)Nc1cccc2cc(Cl)ccc12</smiles>

1a, $\mathrm{R}=\mathrm{H}$, chloroquine 1b, $\mathrm{R}=\mathrm{OH}$, hydroxychloroquine<smiles>Oc1c(Cl)cc(Cl)cc1Sc1cc(Cl)cc(Cl)c1O</smiles>

4, Bithionol<smiles>Nc1nc2c(ccn2[C@H]2C[C@H](O)[C@@H](CO)O2)c(=O)[nH]1</smiles>

2, 2,7-Deaza-2'-C-methyladenosine
3, Gemcitabina<smiles>Nc1ccn([C@@H]2O[C@H](CO)[C@@H](O)C2(F)F)c(=O)n1</smiles><smiles>CC(C)OC(=O)[C@H](C)NP(=O)(OC[C@H]1O[C@@H](N2CCC(=O)NC2=O)[C@](C)(F)[C@@H]1O)Oc1ccccc1</smiles>

Figure 1. Approved drugs that have been used against ZIKV.

the development of a vaccine that may go to human trials in 5 years. ${ }^{9}$

Alternatively, since there is no drug approved for the treatment of Zika infection, drug repurposing screening of pharmaceuticals or biopharmaceuticals as therapeutic or prophylactic agents has recently been explored. ${ }^{10-12}$ There are still no approved drugs for specific therapy to prevent or treat ZIKV infection. ${ }^{13}$ The search for new uses of drugs already on the market can be a good strategy in view of the safety proven mainly in the case of pregnant women. However, the discovery of molecules with different structures and mechanisms of action is also of great importance. Indeed, recently, several drugs have been evaluated against ZIKV with promising results (Figure 1), as for example chloroquine 1a, hydroxychloroquine $\mathbf{1 b},{ }^{14}$ 7-Deaza-2'-C-methyladenosine (2), ${ }^{15,16}$ gemcitabine $(\mathbf{3})^{17}$ and bithionol (4). ${ }^{18}$ Elfiky ${ }^{19}$ compared by molecular docking several inhibitors of the viral polymerases of ZIKV and hepatitis $\mathrm{C}$ virus (HCV) and found ribavirin and sofosbuvir (Figure 1) are possibly effective in regulating the life cycle of these viruses. ${ }^{19}$ More recently, Sacramento et al. ${ }^{20}$ have demonstrated that the antiviral sofosbuvir (5), used against the HCV, which is also in the Flaviviridae, inhibits ZIKV ribonucleic acid (RNA) polymerase, targeting conserved amino acid residues.

Several previous studies ${ }^{21,22}$ indicated that compounds containing a naphthoquinone moiety play key roles in the respiratory cycle, electron transport chain, blood clotting and the carboxylation of glutamate in cells. The mechanism of action of naphthoquinones may involves the generation of reactive oxygen species (ROS) induced by bioreduction of the quinone nucleus by specific enzymes to generate reactive oxygen species inside virus. ${ }^{23}$ Other possible mechanism of action include intercalation, induction of breaks in the deoxyribonucleic acid (DNA) chain and bioreductive alkylation via formation of quinone methide. ${ }^{24}$ Among the compound inserted in the class of naphthoquinones, can be cited the droserone (6) which reduced the production of viral particle of measles virus to $50 \%$ (half maximal effective concentration, $\mathrm{EC}_{50}$ ) at a concentration of $2 \mu \mathrm{M},{ }^{25} 2$-aminomethyl3-hydroxy-1,4-naphthoquinone (7) which showed promising antiviral activity against Herpes simplex virus type-1 (HSV-1) presenting $\mathrm{EC}_{50}$ of $0.83 \mu \mathrm{M}$ and showed activity comparable to acyclovir, the antiviral currently used clinically, which had $\mathrm{EC}_{50}$ of $1.09 \mu \mathrm{M},{ }^{26}$ the pyranaphthoquinone (8) that exhibited $\mathrm{EC}_{50}$ of $0.3114 \mu \mathrm{M}$ for dengue virus replication in mammalian cells ${ }^{27}$ and the naphthoquinone trimer (9) which was inhibitor of hepatitis $\mathrm{B}$ virus (HBV) and had $\mathrm{EC}_{50}$ of $0.009 \mu \mathrm{M}$ and selective index above 3000 (Figure 2). ${ }^{28}$<smiles>CC1=C(O)C(=O)c2c(O)cccc2C1=O</smiles><smiles>O=C1C(O)=C(C(NCc2ccccc2)c2ccc(Cl)cc2Cl)C(=O)c2ccccc21</smiles><smiles>Cc1ccc([C@H]2C[C@H](c3ccc([N+](=O)[O-])cc3)OC3=C2C(=O)C(=O)c2ccccc23)cc1</smiles><smiles>CC1(C)CCc2ccc3c(c2CC1)C(=O)C(=O)C(C1=C(O)C(=O)c2c(ccc4c2CCC(C)(C)O4)C1=O)=C3C(=O)O</smiles>

Figure 2. Examples of antiviral naphthoquinones.

It was shown that bis-hydroxy-1,4-naphthoquinones (10) have promising antimicrobial activity. de Araújo et al. ${ }^{29}$ 
reported that these compounds have moderate activities against Leishmania amazonensis and Leishmania braziliensis promastigotes without significant toxicity. The compound 10a presents high potency against Leishmania amazonensis with $\mathrm{EC}_{50}$ of $0.3 \pm 0.1 \mu \mathrm{M}$ while $10 \mathrm{~b}$ was more active against Leishmania braziliensis with $\mathrm{EC}_{50}$ of $0.9 \pm 0.08 \mu \mathrm{M}$ (Figure 3).

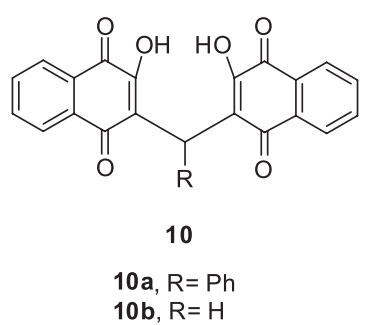

Figure 3. Example of leishmanicidal bis-hydroxy-1,4-naphthoquinones.

The aim of this work is to demonstrate the antiviral potential of bis-naphthoquinones on the replication of ZIKV that has reached a large number of people in the population and has brought serious complications especially in pregnant women.

\section{Experimental}

Chemistry

Reagents were purchased from Sigma-Aldrich and were used without further purification. Column chromatography was performed with silica gel 60 (Merck 70-230 mesh). Analytical thin layer chromatography (TLC) was performed with silica gel plates (Merck, TLC silica gel 60 F254), and the plots were visualized using UV light or aqueous solutions of ammonium sulfate. Yields refer to chromatographically and spectroscopically homogeneous materials.

Melting points were obtained on a Fischer-Johns apparatus and were uncorrected. Infrared spectra were measured using $\mathrm{KBr}$ pellets on a PerkinElmer model 1420 FT-IR spectrophotometer, calibrated relative to the $1601.8 \mathrm{~cm}^{-1}$ absorbance of polystyrene.

Nuclear magnetic resonance (NMR) spectra were recorded on a Varian Unity Plus VXR $(500 \mathrm{MHz})$ instrument in dimethyl sulfoxide (DMSO- $d_{6}$ ) or $\mathrm{CDCl}_{3}$ solutions. The chemical shift data were reported in units of $\delta$ (ppm) downfield from tetramethylsilane or the solvent, either of which were used as an internal standard; coupling constants $(J)$ are reported in hertz and refer to apparent peak multiplicities.

The high-resolution mass spectra (electrospray ionization, HRESIMS) were obtained using a QTOF Micro (Waters) mass spectrometer.
The physical and spectroscopic data for 10a, 10b-e and 10g-o were previously reported by Tisseh and Bazgir, ${ }^{30}$ which developed some efficient method for production bis-benzoquinonylmethanes. ${ }^{31}$

3,3'-(o-Tolylmethylene) bis(2-hydroxynaphthalene1,4-dione) (10f)

Yellow solid; yield $68 \%$; $\mathrm{mp} 177-178^{\circ} \mathrm{C}$; infrared (IR) (KBr) $v / \mathrm{cm}^{-1} 558,579,627,653,644,675,693 ;{ }^{1} \mathrm{H}$ NMR $\left(500 \mathrm{MHz}, \mathrm{DMSO}-d_{6}\right) \delta 8.00(2 \mathrm{H}, \mathrm{dd}, J 7.1$ and $1.7 \mathrm{~Hz})$, $7.95(2 \mathrm{H}, \mathrm{dd}, J 7.1$ and 1.7$), 7.81(2 \mathrm{H}, \mathrm{td}, J 9.0,7.1$ and 1.7), $7.76(2 \mathrm{H}, \mathrm{td}, J 9.0,7.1$ and $1.7 \mathrm{~Hz}), 7.21(1 \mathrm{H}, \mathrm{dd}, J 6.5$ and $2.3 \mathrm{~Hz}), 7.10-7.11(3 \mathrm{H}, \mathrm{m}), 6.05(1 \mathrm{H}, \mathrm{s}), 2.26(1 \mathrm{H}$, s); ${ }^{13} \mathrm{C}$ NMR (125 MHz, DMSO- $\left.d_{6}\right) \delta 19.5,36.44,123.5$, 125.7, 126.0, 126.1, 126.5, 129.2, 129.9, 130.4, 132.7, 133.5, 135.1, 135.9, 139.4, 156.6, 181.5, 183.9; HRMS (electrospray ionization, ESI) $\mathrm{m} / z$, calcd. for $\mathrm{C}_{28} \mathrm{H}_{18} \mathrm{O}_{6} \mathrm{Na}^{+}$ $[\mathrm{M}+\mathrm{Na}]^{+}:$473.1001, found: $473.0980, \Delta=2.1 \mathrm{ppm}$.

General procedure for preparation of bis(naphthalene1,2,4-triyl triacetates) (13a-n)

In a $50 \mathrm{~mL}$ round bottom flask, $1 \mathrm{mmol}$ of bis(2-hydroxynaphthalene-1,4-diones) (12), $7.2 \mathrm{~g}$ of zinc metallic, $15 \mathrm{~mL}$ of acetic anhydride and pyridine catalytic were added. The solution was stirred for $72 \mathrm{~h}$ in room temperature and the progress of the reaction was followed by TLC. In the next step, the solution was filtered, extracted with chloroform and the product was purified by column chromatography, using hexane/ethyl acetate as the eluent.

3,3'-(Phenylmethylene)bis(naphthalene-1,2,4-triyl triacetate) (13a)

White solid; yield 83\%; mp 222-223 ${ }^{\circ} \mathrm{C}$; IR (KBr) $\mathrm{V} / \mathrm{cm}^{-1} 1767,1603,1496,1426,1365,1163,1098$, $1035,908,871,759,733,699,605,585,545 ;{ }^{1} \mathrm{H}$ NMR $\left(500 \mathrm{MHz}, \mathrm{CDCl}_{3}\right) \delta 1.85(\mathrm{~s}, 6 \mathrm{H}), 2.02(\mathrm{~s}, 6 \mathrm{H}), 2.37(\mathrm{~s}$, $6 \mathrm{H}), 6.27(\mathrm{~s}, 1 \mathrm{H}), 7.18-7.24(\mathrm{~m}, 5 \mathrm{H}), 7.46-7.57(\mathrm{~m}, 6 \mathrm{H})$, $7.68(\mathrm{~d}, J 7.8 \mathrm{~Hz}, 2 \mathrm{H}) ;{ }^{13} \mathrm{C} \mathrm{NMR}\left(125 \mathrm{MHz}, \mathrm{CDCl}_{3}\right) \delta 19.9$, 20.5, 20.6, 42.0, 121.7, 121.9, 122.1, 125.6, 126.1, 127.0, $127.1,127.2,127.5,128.7,136.9,139.0,139.1,144.5$, 150.7, 167.0, 168.0, 169.0; HRMS (ESI) $\mathrm{m} / \mathrm{z}$, calcd. for $\mathrm{C}_{39} \mathrm{H}_{32} \mathrm{O}_{12} \mathrm{Na}[\mathrm{M}+\mathrm{Na}]^{+}:$715.1786, found: 715.1792, $\Delta=0.8 \mathrm{ppm}$.

3,3'-(2-Chlorophenylmethylene)bis(naphthalene-1,2,4-triyl triacetate) (13c)

White solid; yield $78 \%$; mp $168-169{ }^{\circ} \mathrm{C}$; IR $(\mathrm{KBr})$ $v / \mathrm{cm}^{-1} 2935,1770,1604,1496,1428,1365,1165$, 1098, 1037, 909, 870, 760, 733, 699, 584, 545; ${ }^{1} \mathrm{H}$ NMR 
$\left(500 \mathrm{MHz}, \mathrm{CDCl}_{3}\right) \delta 1.84(\mathrm{~s}, 6 \mathrm{H}), 1.94(\mathrm{~s}, 6 \mathrm{H}), 2.33(\mathrm{~s}$, $6 \mathrm{H}), 6.53(\mathrm{~s}, 1 \mathrm{H}), 7.11-7.19(\mathrm{~m}, 2 \mathrm{H}), 7.29-7.32(\mathrm{~m}, 2 \mathrm{H})$, 7.41-7.54 (m, 7H), 7.70-7.76 (m, 2H); ${ }^{13} \mathrm{C} \mathrm{NMR}(125 \mathrm{MHz}$, $\left.\mathrm{CDCl}_{3}\right) \delta 19.9,20.6,20.8,40.2,121.9,122.0,122.2,124.8$, 126.0, 127.2, 127.2, 127.5, 127.6, 127.6, 128.6, 129.5, 133.4, 134.9, 136.9, 136.9, 150.3, 166.8, 167.9, 175.6; HRMS (ESI) $\mathrm{m} / z$, calcd. for $\mathrm{C}_{39} \mathrm{H}_{31} \mathrm{ClO}_{12} \mathrm{Na}[\mathrm{M}+\mathrm{Na}]^{+}$: 749.1397, found: $749.1402, \Delta=0.7 \mathrm{ppm}$.

3,3'-(3-Chlorophenylmethylene)bis(naphthalene-1,2,4-triyl triacetate) (13d)

White solid; yield 98\%; mp 167-168 ${ }^{\circ} \mathrm{C}$; IR (KBr) $v / \mathrm{cm}^{-1} 1774,1640,1597,1505,1477,1427,1366$, 1165, 1098, 1037, 1025, 924, 906, 872, 767, 744, 676, 642, 606, 587, 549; ${ }^{1} \mathrm{H}$ NMR (500 MHz, $\left.\mathrm{CDCl}_{3}\right) \delta 1.88$ (s, 6H), $2.03(\mathrm{~s}, 6 \mathrm{H}), 2.34(\mathrm{~s}, 6 \mathrm{H}), 6.19(\mathrm{~s}, 1 \mathrm{H}), 7.14(\mathrm{~s}$, $3 \mathrm{H}), 7.25$ (s, 1H), 7.44-7.55 (m, 6H), $7.74(\mathrm{~d}, J 8.0 \mathrm{~Hz}$, $2 \mathrm{H}) ;{ }^{13} \mathrm{C} \mathrm{NMR}\left(125 \mathrm{MHz}, \mathrm{CDCl}_{3}\right) \delta 19.9,20.6,20.8$, $40.2,121.9,122.0,122.3,124.8,126.0,127.2,127.2$, $127.6,127.6,128.6,129.5,133.4,134.9,136.9$, 136.9, 150.3, 166.8, 167.9, 175.6; HRMS (ESI) $\mathrm{m} / \mathrm{z}$, calcd. for $\mathrm{C}_{39} \mathrm{H}_{31} \mathrm{ClO}_{12} \mathrm{Na}[\mathrm{M}+\mathrm{Na}]^{+}:$749.1402, found: 749.1396, $\Delta=0.8 \mathrm{ppm}$.

3,3'-(4-Chlorophenylmethylene)bis(naphthalene-1,2,4-triyl triacetate) (13e)

White solid; yield 78\%; mp 140-141 ${ }^{\circ} \mathrm{C}$; IR (KBr) $v / \mathrm{cm}^{-1} 1773,1605,1491,1427,1366,1165,1098,1058$, $1025,919,872,829,767,746,678,634,605,586,539$; ${ }^{1} \mathrm{H}$ NMR $\left(500 \mathrm{MHz} \mathrm{CDCl}_{3}\right) \delta 1.87(\mathrm{~s}, 6 \mathrm{H}), 2.03(\mathrm{~s}, 6 \mathrm{H})$, $2.35(\mathrm{~s}, 6 \mathrm{H}), 6.19(\mathrm{~s}, 1 \mathrm{H}), 7.15-7.17(\mathrm{~m}, 4 \mathrm{H}), 7.44-7.55(\mathrm{~m}$, $6 \mathrm{H}), 7.73$ (d, J 8.0 Hz, 2H); $\left.{ }^{13} \mathrm{C} \mathrm{NMR} \mathrm{(125} \mathrm{MHz,} \mathrm{CDCl}_{3}\right)$ $\delta$ 19.7, 20.2, 20.4, 41.1, 121.8, 121.9, 125.0, 125.9, 127.0, $127.1,127.5,128.5,131.0,132.6,136.7,137.6,138.7$, 144.4, 166.8, 167.7, 168.8; HRMS (ESI) $\mathrm{m} / \mathrm{z}$, calcd. for $\mathrm{C}_{39} \mathrm{H}_{31} \mathrm{ClO}_{12} \mathrm{Na}[\mathrm{M}+\mathrm{Na}]^{+}:$749.1404, found: 749.1402 , $\Delta=0.5 \mathrm{ppm}$.

3,3'-(2-Methylphenylmethylene)bis(naphthalene-1,2,4-triyl triacetate) (13f)

White solid; yield $75 \%$; mp $155-157{ }^{\circ} \mathrm{C}$; IR (KBr) $v / \mathrm{cm}^{-1} 1770,1605,1489,1456,1366,1196,1167,1098$, 1057, 1024, 918, 871, 760, 742, 677, 640, 606, 586, 546; ${ }^{1} \mathrm{H}$ NMR $\left(500 \mathrm{MHz}, \mathrm{CDCl}_{3}\right) \delta 1.72(\mathrm{~s}, 6 \mathrm{H}), 1.96(\mathrm{~s}, 6 \mathrm{H})$, $2.12(\mathrm{~s}, 3 \mathrm{H}), 2.33(\mathrm{~s}, 6 \mathrm{H}), 6.22(\mathrm{~s}, 1 \mathrm{H}), 7.03-7.11(\mathrm{~m}$, $3 \mathrm{H}), 7.44-7.53$ (m, 7H), 7.74 (dd, J 12.3 and $4.5 \mathrm{~Hz}, 2 \mathrm{H})$; ${ }^{13} \mathrm{C} \mathrm{NMR}\left(125 \mathrm{MHz}, \mathrm{CDCl}_{3}\right) \delta 19.7,19.8,20.6,20.7,40.2$, $121.9,122.0,122.0,122.2,126.2$, 126.6, 127.0, 127.1, $127.2,127.3,127.5,127.5,130.5,131.6,136.9,136.9$, 167.8; HRMS (ESI) $m / z$, calcd. for $\mathrm{C}_{40} \mathrm{H}_{34} \mathrm{O}_{12} \mathrm{Na}[\mathrm{M}+\mathrm{Na}]^{+}$: 729.1921, found: $729.1948, \Delta=0.9 \mathrm{ppm}$.
3,3'-(3-Methylphenylmethylene)bis(naphthalene-1,2,4-triyl triacetate) $(\mathbf{1 3 g})$

White solid; yield 75\%; mp 245-246 ${ }^{\circ} \mathrm{C}$; IR (KBr) $\mathrm{v} / \mathrm{cm}^{-1} 1763,1606,1425,1366,1195,1168,1099,1027$, 958, 934, 871, 760, 742, 677, 640, 606, 586, 546; ${ }^{1} \mathrm{H}$ NMR $\left(500 \mathrm{MHz}, \mathrm{CDCl}_{3}\right) \delta 1.18(\mathrm{~s}, 6 \mathrm{H}), 1.97(\mathrm{~s}, 6 \mathrm{H}), 2.15(\mathrm{~s}, 3 \mathrm{H})$, $2.29(\mathrm{~s}, 6 \mathrm{H}), 6.16(\mathrm{~s}, 1 \mathrm{H}), 6.89-7.05(\mathrm{~m}, 4 \mathrm{H}), 7.37-7.48(\mathrm{~m}$, $6 \mathrm{H}), 7.76(\mathrm{~d}, J 7.8 \mathrm{~Hz}, 2 \mathrm{H}) ;{ }^{13} \mathrm{C} \mathrm{NMR}\left(125 \mathrm{MHz}, \mathrm{CDCl}_{3}\right)$ $\delta 19.7,20.3,20.4,21.1,41.7,114.5,121.7,121.9,125.6$, $125.9,126.7,126.9,126.9,127.3,127.4,128.4,130.4$, 136.7, 137.9, 138.7, 144.3, 166.6, 167.7, 168.8; HRMS (ESI) $m / z$, calcd. for $\mathrm{C}_{40} \mathrm{H}_{34} \mathrm{O}_{12} \mathrm{Na}[\mathrm{M}+\mathrm{Na}]^{+}:$729.1945, found: $729.1948, \Delta=0.4 \mathrm{ppm}$.

3,3'-(4-Methylphenylmethylene)bis(naphthalene-1,2,4-triyl triacetate) (13h)

White solid; yield 83\%; mp 233-235 ${ }^{\circ} \mathrm{C}$; IR (KBr) $\mathrm{v} / \mathrm{cm}^{-1} 1769,1605,1513,1426,1366,1164,1097,1025$, $918,880,814,758,745,675,605,588,543 ;{ }^{1} \mathrm{H}$ NMR $\left(500 \mathrm{MHz}, \mathrm{CDCl}_{3}\right) \delta 1.83(\mathrm{~s}, 6 \mathrm{H}), 1.99(\mathrm{~s}, 6 \mathrm{H}), 2.26(\mathrm{~s}$, $3 \mathrm{H}), 2.34$ (s, 6H), 6.20 (s, 1H), 6.99 (d, J 8.1 Hz, 2H), 7.09 (2H, d, J 8.1 Hz, 2H), 7.42-7.53 (m, 6H), 7.73 (d, J 7.8 Hz, $2 \mathrm{H}) ;{ }^{13} \mathrm{C} \mathrm{NMR}\left(125 \mathrm{MHz}, \mathrm{CDCl}_{3}\right) \delta 19.7,20.2,20.4,21.0$, 41.5, 121.7, 121.9, 125.7, 125.9, 126.9, 127.3, 129.0, 129.6, 135.7, 136.0, 136.7, 138.8, 144.2, 150.5, 166.7, 167.8, 168.9; HRMS (ESI) $m / z$, calcd. for $\mathrm{C}_{40} \mathrm{H}_{34} \mathrm{O}_{12} \mathrm{Na}[\mathrm{M}+\mathrm{Na}]^{+}$: 729.1932, found: $729.1948, \Delta=0.4 \mathrm{ppm}$.

3,3'-(4-Acetoxyphenylmethylene)bis(naphthalene-1,2,4-triyl triacetate) (13i)

White solid; yield 70\%; mp 285-286 ${ }^{\circ} \mathrm{C}$; IR (KBr) $v / \mathrm{cm}^{-1} 2938,1766,1639,1607,1507,1423,1367,1193$, 1167, 1099, 1026, 943, 923, 908, 884, 767, 746, 684, 637, 605, 588, 549; ${ }^{1} \mathrm{H}$ NMR (500 MHz, $\left.\mathrm{CDCl}_{3}\right) \delta 1.87(\mathrm{~s}, 6 \mathrm{H})$, $2.03(\mathrm{~s}, 6 \mathrm{H}), 2.23(\mathrm{~s}, 3 \mathrm{H}), 2.34(\mathrm{~s}, 6 \mathrm{H}), 6.23$ (s, 1H), $6.90(\mathrm{~d}$, $J 8.7 \mathrm{~Hz}, 2 \mathrm{H}), 7.23$ (d, J 8.7 Hz, 2H), 7.39-7.54 (m, 6H), 7.73 $(\mathrm{d}, J 7.9 \mathrm{~Hz}, 2 \mathrm{H}) ;{ }^{13} \mathrm{C} \mathrm{NMR}\left(125 \mathrm{MHz}, \mathrm{CDCl}_{3}\right) \delta 19.7,20.3$, 20.5, 21.0, 41.3, 121.6, 121.8, 122.0, 125.9, 127.0, 127.4, 130.6, 136.5, 136.7, 138.7, 144.4, 149.5, 166.8, 167.8, 169.0, 169.3; HRMS (ESI) $m / z$, calcd. for $\mathrm{C}_{41} \mathrm{H}_{34} \mathrm{O}_{14} \mathrm{Na}[\mathrm{M}+\mathrm{Na}]^{+}$: 773.1836, found: $773.1847, \Delta=1.4 \mathrm{ppm}$.

3,3'-(3-Acetoxyphenylmethylene)bis(naphthalene-1,2,4-triyl triacetate) (13j)

White solid; yield 82\%; mp 178-179 ${ }^{\circ} \mathrm{C}$; IR (KBr) v / $\mathrm{cm}^{-1}$ 1763, 1606, 1487, 1428, 1365, 1194, 1163, 1098, 1025, 920, 870, 744, 678, 608, 584, 549, 531; ${ }^{1} \mathrm{H}$ NMR (500 MHz, $\left.\mathrm{CDCl}_{3}\right) \delta 1.88(\mathrm{~s}, 6 \mathrm{H}), 2.04(\mathrm{~s}, 6 \mathrm{H}), 2.17(\mathrm{~s}, 3 \mathrm{H}), 2.34(\mathrm{~s}, 6 \mathrm{H})$, 6.25 (s, 1H), 6.90-6.93 (m, 2H), 7.11 (d, J 8.3 Hz, 1H), 7.19 (d, J 8.3 Hz, 1H), 7.43-7.54 (m, 6H), $7.76(\mathrm{~d}, J 7.8 \mathrm{~Hz}, 2 \mathrm{H})$; ${ }^{13} \mathrm{C} \mathrm{NMR}\left(125 \mathrm{MHz}, \mathrm{CDCl}_{3}\right) \delta 19.7,20.3,20.5,21.0,41.3$, 
120.3, 121.8, 122.0, 123.0, 125.0, 125.9, 127.0, 127.4, 129.2, 132.4, 136.7, 138.7, 140.7, 144.5, 150.7, 166.8, 167.8, 169.0, 169.4; HRMS (ESI) $\mathrm{m} / z$, calcd. for $\mathrm{C}_{41} \mathrm{H}_{34} \mathrm{O}_{14} \mathrm{Na}[\mathrm{M}+\mathrm{Na}]^{+}$: 773.1844, found: $773.1847, \Delta=0.4 \mathrm{ppm}$.

3,3 ' - (2, 3, 4, 5, 6-Pentafluorophenylmethylene) bis(naphthalene-1,2,4-triyl triacetate) (13k)

White solid; yield $42 \%$; mp $140-141{ }^{\circ} \mathrm{C}$; IR (KBr) $\mathrm{v} / \mathrm{cm}^{-1}$ 2974, 1779, 1638, 1606, 1523, 1503, 1427, 1370, 1198, 1180, 1100, 1041, 993, 980, 769; ${ }^{1} \mathrm{H}$ NMR $(500 \mathrm{MHz}$, $\left.\mathrm{CDCl}_{3}\right) \delta 1.94(\mathrm{~s}, 6 \mathrm{H}), 2.04(\mathrm{~s}, 6 \mathrm{H}), 2.30(\mathrm{~s}, 6 \mathrm{H}), 6.26$ (s, 1H), 7.42-7.50 (m, 6H), 7.66-7.69 (m, 2H); ${ }^{13} \mathrm{C}$ NMR $\left(125 \mathrm{MHz}, \mathrm{CDCl}_{3}\right) \delta$ 19.7, 20.0, 20.4, 32.0, 114.1, 121.8, $121.9,121.9,122.0,125.6,127.1,127.2,127.7,136.6$, 138.6, 141.4, 144.0, 147.6, 166.9, 167.4, 168.1; HRMS (ESI) $m / z$, calcd. for $\mathrm{C}_{39} \mathrm{H}_{27} \mathrm{~F}_{5} \mathrm{O}_{12} \mathrm{Na}[\mathrm{M}+\mathrm{Na}]^{+}$: 805.1299, found: $805.1321, \Delta=2.7 \mathrm{ppm}$.

3,3'-(4-Bromophenylmethylene)bis(naphthalene-1,2,4-triyl triacetate) (13I)

White solid; yield $60 \%$; mp 296-297 ${ }^{\circ} \mathrm{C}$; IR (KBr) $\mathrm{v} / \mathrm{cm}^{-1}$ 3073, 2974, 2937, 2045, 1777, 1636, 1605, 1504, 1488, 1456, 1425, 1404, 1368, 1310, 1275, 1201, 1193, 1180, 1100, 1072, 1040, 1012, 940, 920, 904, 887, 809, 766, 754, 744; ${ }^{1} \mathrm{H}$ NMR $\left(500 \mathrm{MHz}, \mathrm{CDCl}_{3}\right) \delta 1.82(\mathrm{~s}, 6 \mathrm{H})$, $1.98(\mathrm{~s}, 6 \mathrm{H}), 2.30(\mathrm{~s}, 6 \mathrm{H}), 6.12(1 \mathrm{H}, \mathrm{s}), 7.07(\mathrm{~d}, J 8.7 \mathrm{~Hz}$, $2 \mathrm{H}), 7.27(\mathrm{~d}, J 8.7 \mathrm{~Hz}, 2 \mathrm{H}), 7.39-7.50(\mathrm{~m}, 6 \mathrm{H}), 7.68(\mathrm{~d}$, $J 8.1 \mathrm{~Hz}, 2 \mathrm{H}) ;{ }^{13} \mathrm{C}$ NMR $\left(125 \mathrm{MHz}, \mathrm{CDCl}_{3}\right) \delta 19.8,20.3$, 20.5, 120.7, 121.8, 121.9, 125.0, 125.9, 127.0, 127.1, 127.5, 131.5, 136.8, 138.2, 138.7, 144.4, 166.8, 167.7, 168.8; HRMS (ESI) $m / z$, calcd. for $\mathrm{C}_{39} \mathrm{H}_{31} \mathrm{BrO}_{12} \mathrm{Na}[\mathrm{M}+\mathrm{Na}]^{+}$: 795.0900, found: 795.0897, $\Delta=0.4 \mathrm{ppm}$.

3,3'-(2,4-Dichlorophenylmethylene)bis(naphthalene-1,2,4triyl triacetate) $(\mathbf{1 3 m})$

White solid; yield 97\%; mp 180-181 ${ }^{\circ} \mathrm{C}$; IR (KBr) $\mathrm{v} / \mathrm{cm}^{-1}$ 2973, 2934, 1774, 1637, 1605, 1587, 1559, 1502, 1472, 1452, 1425, 1368, 1276, 1179, 1099, 1040, 918, 876, 825, 769, 751, 708, 676, 641, 607; ' $\mathrm{H}$ NMR (500 MHz, $\left.\mathrm{CDCl}_{3}\right) \delta 1.85(\mathrm{~s}, 6 \mathrm{H}), 1.93(\mathrm{~s}, 6 \mathrm{H}), 2.29(\mathrm{~s}, 6 \mathrm{H}), 6.40(\mathrm{~s}$, $1 \mathrm{H}), 7.07(\mathrm{dd}, J 7.8$ and $1.5 \mathrm{~Hz}, 1 \mathrm{H}), 7.28-7.33(\mathrm{~m}, 2 \mathrm{H})$, 7.48-7.52 (m, 6H), 7.65-7.72 (m, 2H); ${ }^{13} \mathrm{C} \mathrm{NMR}(125 \mathrm{MHz}$, $\left.\mathrm{CDCl}_{3}\right) \delta 19.9,20.0,20.6,39.7,122.0,122.1,122.2,124.4$, 126.0, 127.2, 127.4, 127.7, 127.8, 129.1, 133.8, 134.1, 135.5, 136.0, 136.9, 137.0, 166.6, 167.2, 167.8; HRMS (ESI) $m / z$, calcd. for $\mathrm{C}_{39} \mathrm{H}_{30} \mathrm{Cl}_{2} \mathrm{O}_{12} \mathrm{Na}[\mathrm{M}+\mathrm{Na}]^{+}$: 783.0997, found: $783.1012, \Delta=1.9 \mathrm{ppm}$.

3,3'-(4-Fluorophenylmethylene)bis(naphthalene-1,2,4-triyl triacetate) (13n)

White solid; yield 53\%; mp $167-168{ }^{\circ} \mathrm{C}$; IR (KBr) $\mathrm{v} / \mathrm{cm}^{-1} 1780,1604,1509,1430,1368,1199,1179,1100$, 1041, 919, 876, 836, 768; ${ }^{1} \mathrm{H}$ NMR (500 MHz, $\mathrm{CDCl}_{3}$ ) $\delta 1.82(\mathrm{~s}, 6 \mathrm{H}), 1.99(\mathrm{~s}, 6 \mathrm{H}), 2.29(\mathrm{~s}, 6 \mathrm{H}), 6.14(1 \mathrm{H}, \mathrm{s})$, 7.09-7.12 (m, 4H), 7.38-7.49 (m, 6H), 7.65 (d, J 7.9 Hz, $2 \mathrm{H}) ;{ }^{13} \mathrm{C}$ NMR (125 MHz, $\left.\mathrm{CDCl}_{3}\right) \delta 19.9,20.5,20.6,41.0$, $115.4(\mathrm{~d}, J 21.2 \mathrm{~Hz}), 122.0,122.1,125.4,126.0,127.2$, 127.3, 127.7, 131.6, 135.0, 136.9, 138.9, 144.5, 161.8 (d, $J 244.6 \mathrm{~Hz}$ ), 167.0, 167.8, 169.0; HRMS (ESI) $\mathrm{m} / z$, calcd. for $\mathrm{C}_{39} \mathrm{H}_{31} \mathrm{FO}_{12} \mathrm{Na}[\mathrm{M}+\mathrm{Na}]^{+}:$733.1686, found: 733.1698, $\Delta=1.6 \mathrm{ppm}$.

\section{Cells and viruses}

The African Green monkey kidney cell line (VEROATCCCCL81) obtained from the American Type Collection were grown and maintained in Dulbecco's Modified Eagle Medium (DMEM, Gibco BRL, Life Technologies) containing $50 \mu \mathrm{g} \mathrm{mL}^{-1}$ gentamicin and supplemented with 5\% heat-inactivated and certified Gibco fetal calf serum (FCS). ZIKVMR766 was kindly provided by Dr Davis Fernandes Ferreira, Universidade Federal do Rio de Janeiro, RJ, Brazil.

\section{Cytotoxicity assay}

The cytotoxic effects of all compounds was assayed by (3-(4,5-dimethylthiazol-2-yl)-2,5-diphenyltetrazolium bromide, MTT), ${ }^{32}$ modified by Pinto et al ${ }^{33}$ Vero cells, $3 \times 10^{3} \mathrm{~mL}$, were grown in 96 well microplates at $37^{\circ} \mathrm{C}$ under a $5 \% \mathrm{CO}_{2}$ humidified atmosphere. After $24 \mathrm{~h}$, the cells were treated with different concentrations of compounds $(25,50,100,200$ or $400 \mu \mathrm{M})$ and maintained under the same conditions as above described and untreated Vero cells were used as controls. After $72 \mathrm{~h}$ incubation, the supernatants were discarded and MTT (Sigma Aldrich), $100 \mu \mathrm{L}$ of $5 \mathrm{mg} \mathrm{mL}^{-1}$ in DMEM medium, was added to each well and re-incubated for $3 \mathrm{~h}$ at $37{ }^{\circ} \mathrm{C}$ in $5 \% \mathrm{CO}_{2}$. Then, the medium was discarded and DMSO (100 $\mu \mathrm{L})$ added following re-incubation for another $30 \mathrm{~min}$. Optical densities were measured at $520 \mathrm{~nm}$ on a microplate reader (Thermo Plate TP-Reader). The compound concentration required to reduce the optical density of MTT was calculated by linear regression and results expressed as 50\% cytotoxic concentration $\left(\mathrm{CC}_{50}\right)$. The experiments were performed in quadruplicate and for three times independently.

Screening of antiviral activity of compounds by plaque reduction assay

An antiviral screening test was performed with Vero cells $\left(2 \times 10^{4}\right)$ grown in 24 well microplates inoculated 
with ZIKV (MOI 0.1), as described previously ${ }^{34}$ with some modifications. ${ }^{33}$ After $2 \mathrm{~h}$ virus adsorption at $37{ }^{\circ} \mathrm{C}$ in $5 \%$ $\mathrm{CO}_{2}$, the supernatant was discarded and the cells were washed for removal of the non-adherent viral particles followed by addition of $40 \mu \mathrm{M}$ of different compounds $(10 \mathbf{a}-\mathbf{0})$ and $(13 \mathbf{a}-\mathbf{n})$ in order to identify if any of these compounds had the ability to inhibit the replication of ZIKV. Then, the infected and treated plaques with the respective compounds were re-incubated for $120 \mathrm{~h}$ at $37^{\circ} \mathrm{C}$ in $5 \% \mathrm{CO}_{2}$. The cell monolayer was then fixed and stained with $1 \%$ crystal violet in $10 \%$ formalin. The compound concentration required to reduce the viral plaque number by $50 \%\left(\mathrm{EC}_{50}\right)$ in the treated cells compared to untreated ones was performed as described by Pinto et al..$^{33}$

\section{Extraction and quantification of viral RNA by real time-PCR}

The compounds which showed high inhibition of viral replication were subjected to the real-time polymerase chain reaction $(\mathrm{PCR})$ assay. For this, $5 \times 10^{5}$ Vero cells were grown in 24-well plates in DMEM medium supplemented with 5\% fetal bovine serum (FBS) and subsequently infected with ZIKV (MOI 0.1) for $1 \mathrm{~h}$. After this time, the cells were washed and the compounds $100,13 \mathbf{e}, \mathbf{1 3 h}, \mathbf{1 3} \mathbf{j}$ and $\mathbf{1 3 k}$ were added at concentrations of $1.25,2.5,5.0$ or $10 \mu \mathrm{M}$ and incubated for four days. Subsequently, the supernatants were collected for the extraction of viral RNA by Bio Gene Viral DNA/RNA Extraction, as described by Ogura et al. ${ }^{35}$ The real time PCR
(RT-PCR) was performed using the Bio Gene Zika Virus PCR kit in the CFX96 ${ }^{\mathrm{TM}}$ IVD Real-Time PCR system. The results were evaluated taking into consideration the viral RNA quantification in relation to the triplicate positive control of three independent experiments. To determine the inhibition characteristic of the compounds, we used Ribavirin as a control.

\section{Statistical analysis}

The data were analyzed by the Tukey's test using the GraphPad Instat version 3 program. ${ }^{36} \mathrm{~A} p$ value of $<0.05$ was considered statistically significant.

\section{Results}

\section{Synthesis}

The bis-2-hydroxynaphthalene-1,4-diones or bishydroxy-1,4-naphthoquinones (10) can be easily prepared by reaction of 2 equivalents of lawsone (11) and arylaldehydes (12) (Scheme 1), using the procedure described by Tisseh and Bazgir, ${ }^{30}$ with yields ranging from $50-98 \%$. In the next step the bis(naphthalene-1,2,4-triyl triacetates) (13a-n) were prepared by one-pot reductive acetylation of 10a-n in the presence of zinc metallic, acetic anhydride and pyridine as a catalyst with yields ranging from $42-97 \%$.

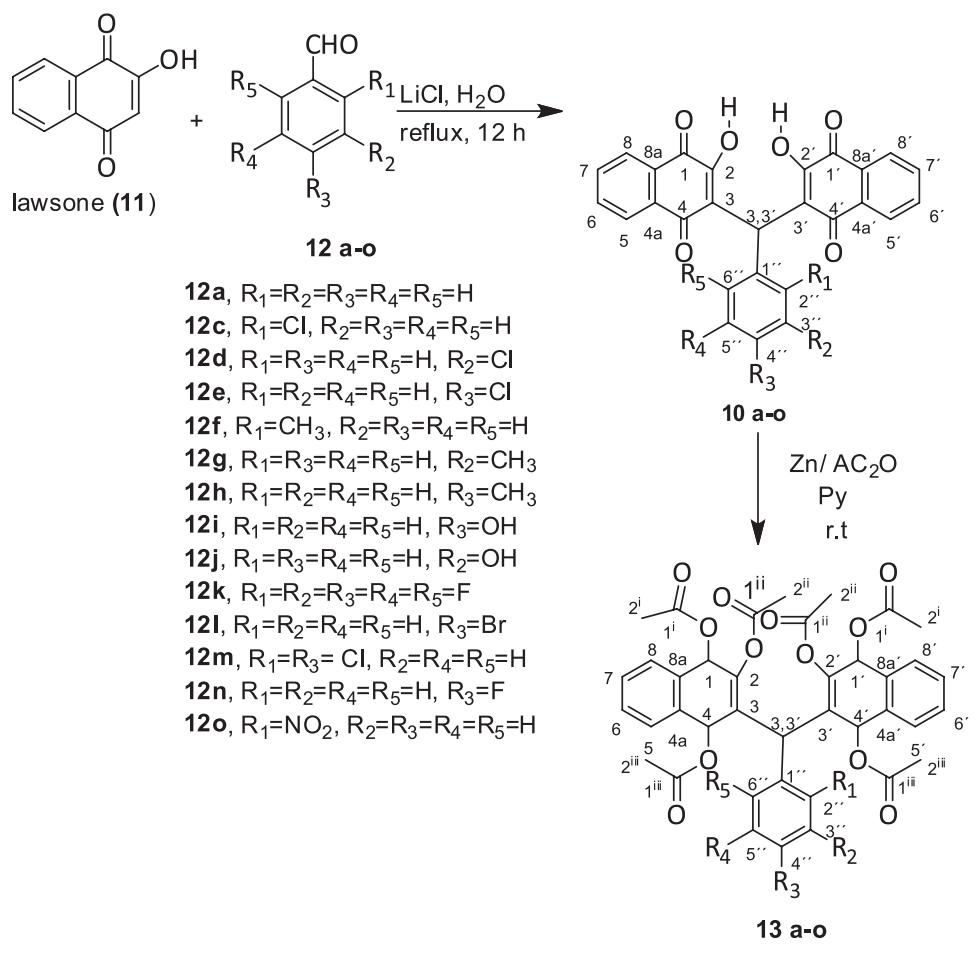

10a, $\mathrm{R}_{1}=\mathrm{R}_{2}=\mathrm{R}_{3}=\mathrm{R}_{4}=\mathrm{R}_{5}=\mathrm{H}, 83 \%$

10c, $R_{1}=C I, R_{2}=R_{3}=R_{4}=R_{5}=H, 90 \%$

$10 \mathrm{~d}, \mathrm{R}_{1}=\mathrm{R}_{3}=\mathrm{R}_{4}=\mathrm{R}_{5}=\mathrm{H}, \mathrm{R}_{2}=\mathrm{Cl}, 98 \%$

10e, $R_{1}=R_{2}=R_{4}=R_{5}=H, R_{3}=C l, 83 \%$

10f, $\mathrm{R}_{1}=\mathrm{CH}_{3}, \mathrm{R}_{2}=\mathrm{R}_{3}=\mathrm{R}_{4}=\mathrm{R}_{5}=\mathrm{H}, 68 \%$

$10 \mathrm{~g}, \mathrm{R}_{1}=\mathrm{R}_{3}=\mathrm{R}_{4}=\mathrm{R}_{5}=\mathrm{H}, \mathrm{R}_{2}=\mathrm{CH}_{3}, 93 \%$

10h, $R_{1}=R_{2}=R_{4}=R_{5}=H, R_{3}=C_{3}, 90 \%$

$10 \mathrm{i}, \mathrm{R}_{1}=\mathrm{R}_{2}=\mathrm{R}_{4}=\mathrm{R}_{5}=\mathrm{H}, \mathrm{R}_{3}=\mathrm{OH}, 87 \%$

$10 \mathrm{j}, \mathrm{R}_{1}=\mathrm{R}_{3}=\mathrm{R}_{4}=\mathrm{R}_{5}=\mathrm{H}, \mathrm{R}_{2}=\mathrm{OH}, 65 \%$

$10 k, R_{1}=R_{2}=R_{3}=R_{4}=R_{5}=F, 65 \%$

10I, $R_{1}=R_{2}=R_{4}=R_{5}=H, R_{3}=B r, 87 \%$

$10 \mathrm{~m}, \mathrm{R}_{1}=\mathrm{R}_{3}=\mathrm{Cl}, \mathrm{R}_{2}=\mathrm{R}_{4}=\mathrm{R}_{5}=\mathrm{H}, 85 \%$

10n, $R_{1}=R_{2}=R_{4}=R_{5}=H, R_{3}=F, 50 \%$

10o, $R_{1}=N_{2}, R_{2}=R_{3}=R_{4}=R_{5}=H, 80 \%$

13a, $R_{1}=R_{2}=R_{3}=R_{4}=R_{5}=H, 83 \%$

13c, $R_{1}=C l, R_{2}=R_{3}=R_{4}=R_{5}=H, 78 \%$

13d, $R_{1}=R_{3}=R_{4}=R_{5}=H, R_{2}=C l, 98 \%$

13e, $R_{1}=R_{2}=R_{4}=R_{5}=H, R_{3}=C l, 78 \%$

13f, $\mathrm{R}_{1}=\mathrm{CH}_{3}, \mathrm{R}_{2}=\mathrm{R}_{3}=\mathrm{R}_{4}=\mathrm{R}_{5}=\mathrm{H}, 75 \%$

13g, $\mathrm{R}_{1}=\mathrm{R}_{3}=\mathrm{R}_{4}=\mathrm{R}_{5}=\mathrm{H}, \mathrm{R}_{2}=\mathrm{CH}_{3}, 75 \%$

13h, $R_{1}=R_{2}=R_{4}=R_{5}=H, R_{3}=C_{3}, \quad 83 \%$

13i, $R_{1}=R_{2}=R_{4}=R_{5}=H, R_{3}=O H, 70 \%$

13j, $R_{1}=R_{3}=R_{4}=R_{5}=H, R_{2}=O H, 82 \%$

13k, $R_{1}=R_{2}=R_{3}=R_{4}=R_{5}=F, 42 \%$

13I, $R_{1}=R_{2}=R_{4}=R_{5}=H, R_{3}=B r, 60 \%$

$13 \mathrm{~m}, \mathrm{R}_{1}=\mathrm{R}_{3}=\mathrm{Cl}, \mathrm{R}_{2}=\mathrm{R}_{4}=\mathrm{R}_{5}=\mathrm{H}, 97 \%$

13n, $R_{1}=R_{2}=R_{4}=R_{5}=H, R_{3}=F, 53 \%$

Scheme 1. Synthetic route to prepare compounds $\mathbf{1 0}$ and $\mathbf{1 3 .}$ 
The aim of this study was to screen the bisnaphthoquinones series $\mathbf{1 0}$ and $\mathbf{1 3}$ for their potential activity against ZIKV.

\section{Evaluation of the anti-ZIKV activity of the compounds}

To observe the toxicity of the naphthoquinones compounds studied, MTT assays were performed in Vero cells using concentrations of the compounds ranging from 25 to $400 \mu \mathrm{M}$ and incubated for 24 to $48 \mathrm{~h}$ to analyze the cytopathic effect. The results in Table 1 show that the series of thirteen compounds (10a-o) have $\mathrm{CC}_{50}$ values from 2876 to $992 \mu \mathrm{M}$. However, the series with fourteen compounds (13a-n) showed lower $\mathrm{CC}_{50}$ values from 549 to $63 \mu \mathrm{M}$,

Table 1. Cytotoxicity $\left(\mathrm{CC}_{50}\right)$ and anti-ZIKV profile (PI: percentage of inhibition ZIKV replication) of compounds of series $\mathbf{1 0}$ and $\mathbf{1 3}$

\begin{tabular}{|c|c|c|}
\hline Compound & $\mathrm{CC}_{50}{ }^{\mathrm{a}} / \mu \mathrm{M}$ & $\mathrm{PI}^{\mathrm{b}} / \%$ \\
\hline $10 \mathrm{a}$ & 981 & 0 \\
\hline $10 \mathrm{c}$ & 1255 & 0 \\
\hline 10d & 1325 & 0 \\
\hline $10 \mathrm{e}$ & 1721 & 0 \\
\hline $10 f$ & 1103 & 0 \\
\hline $10 \mathrm{~g}$ & 1482 & 80 \\
\hline $10 \mathrm{~h}$ & 1465 & 0 \\
\hline $10 \mathrm{i}$ & 2128 & 0 \\
\hline $10 \mathrm{j}$ & 2876 & 0 \\
\hline $10 \mathrm{k}$ & 992 & 0 \\
\hline 101 & 1176 & 0 \\
\hline $10 \mathrm{~m}$ & 1604 & 0 \\
\hline $10 n$ & 1635 & 50 \\
\hline 100 & 2297 & $>99$ \\
\hline $13 a$ & 527 & 0 \\
\hline $13 c$ & 278 & 60 \\
\hline 13d & 428 & 70 \\
\hline $13 e$ & 483 & $>99$ \\
\hline $13 f$ & 121 & 55 \\
\hline $13 \mathrm{~g}$ & 549 & 0 \\
\hline $13 \mathrm{~h}$ & 505 & $>99$ \\
\hline $13 \mathbf{i}$ & 231 & 0 \\
\hline $13 \mathrm{j}$ & 132 & $>99$ \\
\hline $13 k$ & 63 & $>99$ \\
\hline 131 & 130 & 0 \\
\hline $13 m$ & 238 & 0 \\
\hline $13 n$ & 365 & 0 \\
\hline
\end{tabular}

${ }^{a}$ Concentration that reduced $50 \%$ cytotoxic when compared to untreated controls; ${ }^{b} \mathrm{PI}$ : percentage of ZIKV inhibition at $40 \mu \mathrm{M}$ of compounds. The mean values \pm standard deviations are representative of three independent experiments. due to the acetylation of the molecules. In this way, we identified those compounds which to perform the antiviral assays at concentrations well below the toxic concentration while maintaining approximately $>99 \%$ of cell viability.

The compounds with the most promising anti-ZIKV effects were found to be $\mathbf{1 0 o}, \mathbf{1 3 e}, \mathbf{1 3 h}, \mathbf{1 3} \mathbf{j}$ and $\mathbf{1 3} \mathbf{k}$ which were able to inhibit $>99 \%$ ZIKV replication (Table 1).

The naphthoquinone derivatives $(\mathbf{1 0 o}, \mathbf{1 3 e}, \mathbf{1 3} \mathbf{h}, \mathbf{1 3} \mathbf{j}$ and 13k) which showed $100 \%$ inhibition (Table 1) were also evaluated by RT-PCR on infected and treated cells. These compounds were chosen to be used in the determination of the mechanism of ZIKV inhibition and were selected to perform RT-PCR studies in a dose response curve, with concentrations ranging from 1.25 to $10 \mu \mathrm{M}$ (Figure 4).

The results showed that the compounds were still capable of inhibiting the replication of ZIKV by more than $90 \%$ with the lower concentration tested confirming our previous findings, except for the concentration 13k at $1.25 \mu \mathrm{M}$.

After determining the inhibitory potential of the compounds, the plaque reduction assays of the $\mathrm{EC}_{50}$ values (Table 2) were investigated in Vero cell culture. Different compound concentrations were tested (from 0.65 to $20 \mu \mathrm{M}$ ) for $\mathbf{1 0 o}, \mathbf{1 3 e}, \mathbf{1 3} \mathbf{h}, \mathbf{1 3} \mathbf{j}$ and $\mathbf{1 3 k}$, which showed the greatest inhibitory potential for ZIKV replication. The results demonstrate that all derivatives showed potent inhibition of ZIKV replication with $\mathrm{EC}_{50}$ values ranging from 0.62 to $1.38 \mu \mathrm{M}$. However, when cytotoxicity is considered, the $\mathbf{1 0 0}$ derivative gave the highest result with a selectivity index (SI) value of $1664 \mu \mathrm{M}$. Ribavirin was used as control and presented an $\mathrm{EC}_{50}$ of $2.98 \mu \mathrm{M}$.

\section{Discussion}

Several research groups are seeking new compounds to inhibit ZIKV infection, which has been a major public health problem since the outbreak of severe cases in the February 2016, with this virus as an important public health emergency of international concern, a large amount of data was generated in ZIKV infections. This includes details of the pathogenesis of ZIKV, transmission routes, diagnosis, vaccines and antiviral development. ${ }^{37}$ The long persistence of ZIKV infection, lasting for a few weeks to months, and the decrease in viremia emphasis the urgent need of studies of substances with antiviral potential against these arboviruses. ${ }^{38,39}$

In the present study, the inhibitory potential of naphthoquinone-derived compounds on ZIKV virus replication was evaluated. Initially, studies of the inhibition of viral replication in Vero cells using $20 \mu \mathrm{M}$ concentrations of a panel of twenty seven naphthoquinone derivatives 

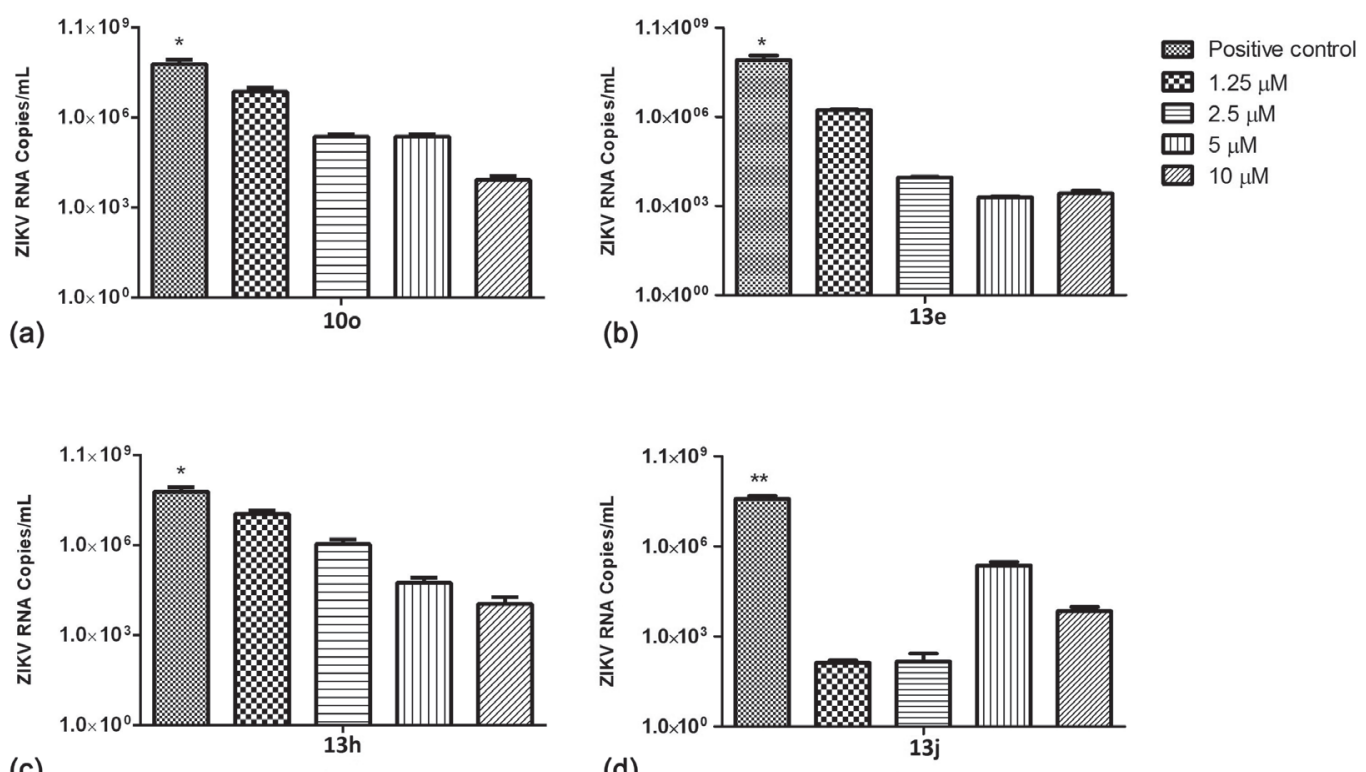

(c)

(d)

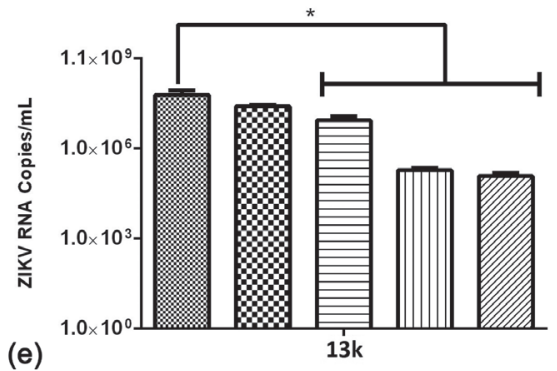

Figure 4. Effect of compounds 100 (a), 13e (b), 13h (c) 13j (d) and 13k (e) at concentrations of 1.25, 2.5, 5 and $10 \mu \mathrm{M}$ on ZIKV genomic RNA by quantitative PCR. Statistical analysis was performed using Tukey's test. ${ }^{*} p<0.05$, ${ }^{* *} p<0.01$.

Table 2. Cytotoxicity $\left(\mathrm{CC}_{50}\right)$, anti-ZIKV profile $\left(\mathrm{EC}_{50}\right)$ and selectivity index (SI) for compounds $10 \mathbf{1 0}, \mathbf{1 3 e}, \mathbf{1 3 h}, \mathbf{1 3} \mathbf{j}$ and $\mathbf{1 3 k}$

\begin{tabular}{lccc}
\hline Compound & $\mathrm{CC}_{50}{ }^{\mathrm{a}} / \mu \mathrm{M}$ & $\mathrm{EC}_{50}{ }^{\mathrm{b}} / \mu \mathrm{M}$ & $\mathrm{SI}^{\mathrm{c}}$ \\
\hline $\mathbf{1 0 0}$ & 2297 & 1.38 & 1664 \\
$\mathbf{1 3 e}$ & 483 & 0.65 & 743 \\
$\mathbf{1 3 h}$ & 505 & 1.11 & 454 \\
$\mathbf{1 3 j}$ & 132 & 0.62 & 213 \\
$\mathbf{1 3 k}$ & 63 & 0.91 & 69 \\
\hline
\end{tabular}

${ }^{\mathrm{a} C}$ Concentration that reduced $50 \%$ cytotoxic when compared to untreated controls; ${ }^{b}$ concentration that reduced $50 \%$ of ZIKV replication when compared to infected controls; ' selectivity index was defined as the ratio between $\mathrm{CC}_{50}$ and $\mathrm{EC}_{50}$ and represents the safety for in vitro assays. The mean values \pm standard deviations are representative of three independent experiments.

was undertaken and the results identified five derivatives able to inhibit $>99 \%$ ZIKV replication. Considering that currently there are no drugs approved for the treatment of ZIKV infection ${ }^{40}$ it is essential to study compounds with high capacity to inhibit viral replication and that have low toxicity may be a good strategy to reduce morbidity and mortality in cases of ZIKV infection. Studies to evaluate the true inhibition potential were initiated with dosedependent response tests using different concentrations of the derivatives in Veri cells infected with ZIKV, aiming the analysis of the mechanism of action. ${ }^{41,42}$ The results demonstrated that the naphthoquinone compounds are able to inhibit viral replication by $50 \%$ at very low doses. For example, compound $\mathbf{1 0 o}$ has an $\mathrm{EC}_{50}$ of $1.38 \mu \mathrm{M}$ while compound 13e has an $\mathrm{EC}_{50}$ in a nanomolar concentration of $0.65 \mu \mathrm{M}$, demonstrating their great potential to inhibit ZIKV. Considering that Ribavirin has been widely used by several groups as a positive control and has been presented as a possible treatment strategy for arbovirus infection, bisnaphthoquinones have an even more potent inhibition profile.

The present study results on the cytotoxicity of the naphthoquinones showed that the non-acetylated derivatives had lower cytotoxicities than the acetylated derivatives with some of the former compounds having 4 times less cytotoxicity than the latter (Table 1). However, in contrast to non-acetylated compounds which are less toxic, we observed that a greater number of acetylates 
showed a high capacity to inhibit the replication of ZIKV. Moreira et al..$^{43}$ identified acetylated isolapachol (14) as a potent and selective antiplasmodium agent, corroborating to the idea that acetylation may be one of the factors increasing biological activity (Figure 5).

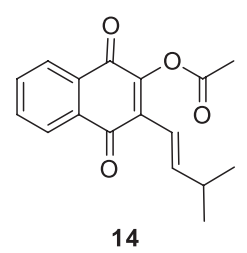

Figure 5. Acetylated isolapachol (14) with antiplasmodium activity. ${ }^{43}$

There are currently no drugs approved for the treatment of ZIKV-infections, not even for reduce the neuronal damage possibly suffered due to infection. ${ }^{44} \mathrm{~A}$ previous study ${ }^{11}$ also describe several other compounds having promising effects against ZIKV replication, but more time is required for defining and analyzing the mechanisms involved. Furthermore, Barrows et al. ${ }^{13}$ examined 774 Food Drug Administration (FDA)-approved drugs for anti-ZIKV activity and demonstrated that at least 20 tested positive for viral inhibition, including mycophenolate mofetil (15) and sertraline (16), which reduced viral infection in vitro (Figure 6).

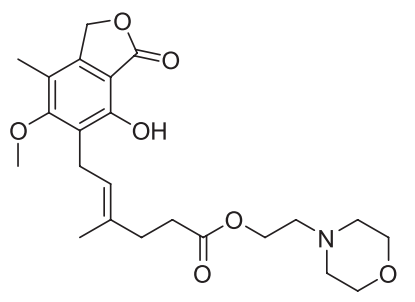

15

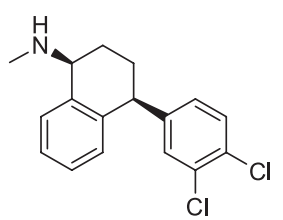

16
Figure 6. Compounds mycophenolate mofetil (15) and sertraline (16) with effects against ZIKV.45

The guaranteed standard for using a drug for antiviral therapy is in its selectivity index (SI), which consists of the ratio between a $50 \%$ cytotoxic concentration $\left(\mathrm{CC}_{50}\right)$ of the cells and the $50 \%$ inhibitory concentration $\left(\mathrm{EC}_{50}\right)$ of the virus. The ideal drug should have an SI between 100 and 1000. The results demonstrate that the $\mathrm{CC}_{50}$ values combined with the $\mathrm{EC}_{50}$ values generate an index above 100 for almost all the compounds except for $\mathbf{1 3 k}$. As can be seen in Table 2, compound 10o showed a higher SI $1664 \mu \mathrm{M}$ followed by 13e which, although much lower, still had a SI of $743 \mu \mathrm{M}$.

The effects of the naphthoquinone derivatives evaluated by RT-PCR on infected and treated viral cells support the idea that the ability to inhibit viral replication is not only to reduce the number of viral particles produced, as assessed by the viral plaques assay, low concentrations of the compounds were able to significantly inhibit the replication of the ZIKV particles. Additionally we have shown that the compounds were also able to inhibit viral RNA synthesis. Therefore, several workers have identified compounds capable of inhibiting the replication of ZIKV by different mechanisms of action ${ }^{45}$ but studies are required to detect compounds not only capable of inhibiting viral replication but also with the ability of reducing morbidity and mortality in cases of new outbreaks.

\section{Conclusions}

Our findings showed that among the naphthoquinone compounds analyzed, five were particularly promising. Compound 10o with $o$-nitro showed the best selectivity index, followed by compound 13e with chlorophenylmethylene radical. The antiviral activity observed may give us the hope that these compounds can be exploited therapeutically for new urgently needed antiviral therapies and their effects will be tested in rodent models for ZIKV infection therapy.

\section{Supplementary Information}

Supplementary information is available free of charge at http://jbcs.sbq.org.br as PDF file.

\section{Acknowledgments}

The authors thank the Brazilian agencies CNPq, CAPES, and FAPERJ and FIOCRUZ for the HRMS. V. F. F., F. C. S. and I. C. N. P. P. are recipients of CNPq research fellowships; D. T. G. G., R. K. F. M. and N. A. R. received FAPERJ research fellowships. C. C. C. S. thanks to CAPES for the Postdoc fellowship and C. S. B. thanks FAPERJ for the Postdoc fellowship. The authors would like to acknowledge for the technical support of Thereza Elizabeth P. P. Garcia.

\section{References}

1. Sikka, V.; Chattu, V. K.; Popli, R. K.; Galwankar, S. C.; Kelkar, D.; Sawicki, S. G.; Stawicki, S. P.; Papadimos, T. J.; J. Global Infect. Dis. 2016, 8, 3.

2. Rodrigues, L. C.; Lancet 2016, 387, 2070.

3. Mlakar, J.; Korva, M.; Tul, N.; Popović, M.; Poljšak-Prijatelj, M.; Mraz, J.; Kolenc, M.; Resman Rus, K.; Vipotnik, T. V.; Vodušek, V. F.; N. Engl. J. Med. 2016, 2016, 951.

4. Cao-Lormeau, V.-M.; Blake, A.; Mons, S.; Lastère, S.; Roche, C.; Vanhomwegen, J.; Dub, T.; Baudouin, L.; Teissier, A.; Larre, P.; Lancet 2016, 387, 1531. 
5. Dick, G.; Kitchen, S.; Haddow, A.; Trans. R. Soc. Trop. Med. Hyg. 1952, 46, 509.

6. Rasmussen, S. A.; Jamieson, D. J.; Honein, M. A.; Petersen, L. R.; N. Engl. J. Med. 2016, 374, 1981.

7. Malone, R. W.; Homan, J.; Callahan, M. V.; Glasspool-Malone, J.; Damodaran, L.; Schneider, A. D. B.; Zimler, R.; Talton, J.; Cobb, R. R.; Ruzic, I.; PLoS Neglected Trop. Dis. 2016, 10, e0004530.

8. Azarudeen, R. M. S. T.; Govindarajan, M.; AlShebly, M. M.; AlQahtani, F. S.; Amsath, A.; Benelli, G.; J. Cluster Sci. 2016, $20,359$.

9. http://g1.globo.com/bemestar/noticia/2016/01/institutobutantan-espera-ter-vacina-contra-zika-em-ate-5-anos.html, accessed in March 21, 2017.

10. Xu, M.; Lee, E. M.; Wen, Z.; Cheng, Y.; Huang, W.-K.; Qian, X.; Julia, T.; Kouznetsova, J.; Ogden, S. C.; Hammack, C.; Nat. Med. 2016, 10, 1101.

11. Adcock, R. S.; Chu, Y.-K.; Golden, J. E.; Chung, D.-H.; Antiviral Res. 2017, 138, 47.

12. Balasubramanian, A.; Teramoto, T.; Kulkarni, A. A.; Bhattacharjee, A. K.; Padmanabhan, R.; Antiviral Res. 2017, 137, 141.

13. Barrows, N. J.; Campos, R. K.; Powell, S. T.; Prasanth, K. R.; Schott-Lerner, G.; Soto-Acosta, R.; Galarza-Muñoz, G.; McGrath, E. L.; Urrabaz-Garza, R.; Gao, J.; Cell Host Microbe 2016, 20, 259.

14. Shiryaev, S. A.; Mesci, P.; Pinto, A.; Fernandes, I.; Sheets, N.; Shresta, S.; Farhy, C.; Huang, C.-T.; Strongin, A. Y.; Muotri, A. R.; Sci. Rep. 2017, 7, 15771.

15. Zmurko, J.; Marques, R. E.; Schols, D.; Verbeken, E.; Kaptein, S. J.; Neyts, J.; PLoS Neglected Trop. Dis. 2016, 10, e0004695.

16. Eyer, L.; Nencka, R.; Huvarová, I.; Palus, M.; Alves, M. J.; Gould, E. A.; de Clercq, E.; Růžek, D.; J. Infect. Dis. 2016, $214,707$.

17. Kuivanen, S.; Bespalov, M. M.; Nandania, J.; Ianevski, A.; Velagapudi, V.; de Brabander, J. K.; Kainov, D. E.; Vapalahti, O.; Antiviral Res. 2016, 139, 117.

18. Leonardi, W.; Zilbermintz, L.; Cheng, L. W.; Zozaya, J.; Tran, S. H.; Elliott, J. H.; Polukhina, K.; Manasherob, R.; Li, A.; Chi, X.; Sci. Rep. 2016, 6, 34475.

19. Elfiky, A. A.; J. Med. Virol. 2016, 88, 2044.

20. Sacramento, C. Q.; de Melo, G. R.; de Freitas, C. S.; Rocha, N.; Hoelz, L. V. B.; Miranda, M.; Fintelman-Rodrigues, N.; Marttorelli, A.; Ferreira, A. C.; Barbosa-Lima, G.; Sci. Rep. 2017, 7, 40920.

21. O'brien, P.; Chem.-Biol. Interact. 1991, 80, 1.

22. da Silva, F. C.; Ferreira, V. F.; Curr. Org. Synth. 2016, 13, 334.

23. Silva, T. L.; Ferreira, F. R.; de Vasconcelos, C. C.; da Silva, R. C.; Lima, D. J. P.; Costa, P. R.; Netto, C. D.; Goulart, M. O.; ChemElectroChem 2016, 3, 2252.

24. Cao, S.; Peng, X.; Curr. Org. Chem. 2014, 18, 70.
25. Lieberherr, C.; Zhang, G.; Grafen, A.; Singethan, K.; Kendl, S.; Vogt, V.; Maier, J.; Bringmann, G.; Schneider-Schaulies, J.; Planta Med. 2017, 83, 232.

26. Pires, M. C.; Sardoux, N.; Terra, L.; Amorim, L.; Vargas, M.; da Silva, G.; Castro, H.; Giongo, V.; Madeira, L.; Paixão, I.; Antiviral Ther. 2015, 21, 507.

27. da Costa, E. C.; Amorim, R.; da Silva, F. C.; Rocha, D. R.; Papa, M. P.; de Arruda, L. B.; Mohana-Borges, R.; Ferreira, V. F.; Tanuri, A.; da Costa, L. J.; PloS One 2013, 8, e82504.

28. Crosby, I. T.; Bourke, D. G.; Jones, E. D.; Jeynes, T. P.; Cox, S.; Coates, J. A.; Robertson, A. D.; Bioorg. Med. Chem. Lett. 2011, 21, 1644.

29. de Araújo, M. V.; de Souza, P. S.; de Queiroz, A. C.; da Matta, C. B.; Leite, A. B.; da Silva, A. E.; de França, J. A.; Silva, T.; Camara, C. A.; Alexandre-Moreira, M. S.; Molecules 2014, 19, 15180.

30. Tisseh, Z. N.; Bazgir, A.; Dyes Pigm. 2009, 83, 258.

31. Brahmachari, G.; ACS Sustainable Chem. Eng. 2015, 3, 2058.

32. Mosmann, T.; J. Immunol. Methods 1983, 65, 55.

33. Pinto, A. M. V.; Leite, J. P. G.; Neves, A. P.; da Silva, G. B.; Vargas, M. D.; Paixão, I. C. N.; Arch. Virol. 2014, 159, 1827.

34. Cheng, H.-Y.; Lin, T.-C.; Yang, C.-M.; Wang, K.-C.; Lin, L.-T.; Lin, C.-C.; J. Antimicrob. Chemother. 2004, 53, 577.

35. Ogura, T.; Tsuchiya, A.; Minas, T.; Mizuno, S.; BMC Res. Notes 2015, 8, 644.

36. GraphPad Instat version 3; GraphPad Software Inc; San Diego, CA, USA, 2002.

37. Musso, D.; Nilles, E.; Cao Lormeau, V. M.; Clin. Microbiol. Infect. 2014, 20, O595.

38. Musso, D.; Roche, C.; Robin, E.; Nhan, T.; Teissier, A.; CaoLormeau, V.-M.; Emerging Infect. Dis. 2015, 21, 359.

39. Campos, R. M.; Cirne-Santos, C.; Meira, G. L.; Santos, L. L.; de Meneses, M. D.; Friedrich, J.; Jansen, S.; Ribeiro, M. S.; da Cruz, I. C.; Schmidt-Chanasit, J.; J. Clin. Virol. 2016, 77, 69.

40. McArthur, M. A.; Viruses 2017, 9, 143.

41. Pitts, J. D.; Li, P. C.; de Wispelaere, M.; Yang, P. L.; Antiviral Res. 2017, 147, 124.

42. Vazquez-Calvo, A.; Jimenez de Oya, N.; Martin-Acebes, M. A.; Garcia-Moruno, E.; Saiz, J. C.; Front. Microbiol. 2017, 8, 1314.

43. Moreira, D. R.; de Sá, M. S.; Macedo, T. S.; Menezes, M. N.; Reys, J. R. M.; Santana, A. E.; Silva, T. L.; Maia, G. L.; Barbosa-Filho, J. M.; Camara, C. A.; J. Enzyme Inhib. Med. Chem. 2015, 30, 615.

44. Salam, A. P.; Rojek, A.; Dunning, J.; Horby, P. W.; Ann. Intern. Med. 2017, 166, 725.

45. Ali, A.; Wahid, B.; Rafique, S.; Idrees, M.; Asian Pac. J. Trop. Med. 2017, 10, 321.

Submitted: December 12, 2018

Published online: April 25, 2019 